\title{
Effect of chiropractic and lumbar exercise program on lumbar muscle strength and Cobb's angle in patients with scoliosis for u-Healthcare
}

\author{
Minwoo Cheon ${ }^{1}$, Jaeyong Park ${ }^{2}$, Yangsun Lee ${ }^{3}$ and Jungchul Lee ${ }^{4^{*}}$
}

\begin{abstract}
This study aimed to find the effects of 12-week chiropractic and lumbar exercise program on the lumbar muscle strength and Cobb's angle in 16 adolescent students with scoliosis. The patients with scoliosis did not have neurological symptoms and surgery experience. Each group of eight subjects received chiropractic and lumbar exercises three times a week. Subjects' lumbar muscle strength measurement showed a statistically significant temporal difference in the chiropractic group (CG) and chiropractic with exercise group (EG) and had interaction effects. However, no significant difference was found between the two groups. Changes in the Cobb's angle in each group had a significant difference both temporally and between group in both CG and EG, and interaction effects were also significant. As suggested in this study, mutual application of exercise therapy program and chiropractic treatments was found to have a positive effect on lumbar muscle strength and Cobb's angle in patients with scoliosis.
\end{abstract}

Keywords: Scoliosis, Chiropractic, Lumbar exercise, Cobb's angle

\section{Introduction}

Human joints are continually connected to each other; thus, structural or functional changes in one joint immediately affect the connecting joints functionally. When spinal joints are morphologically deformed due to lack of exercise and limited physical activity, the musculoskeletal system will have structural and functional damages, and these damages not only cause exterior postural changes but also internal disorders and pain [1]. Scoliosis is a typical spine disease, and the term was first used by Galen (AD 131-201). Scoliosis is defined as having more than one or two vertebra deviated sideways or rotated $[2,3]$. The cause of scoliosis is unclear but is reported to be the most representative spinal curvature disorder in children [4]. When Cobb's angle is about $10^{\circ}$, thoracic elasticity and vital capacity decrease [5]. When scoliosis is developed by incorrect posture, it can lead to trauma such as back pain, sciatic nerve pain, and disc herniation. These conditions become the direct cause of structural diseases

\footnotetext{
*Correspondence: channel365@hanmail.net

${ }^{4}$ Department of Exercise Prescription, Dongshin University, 252 Daeho-dong, NajuJeonnam 520-714, Republic of Korea

Full list of author information is available at the end of the article
}

including sensory disorder in the extremities, muscular atrophy, chronic dyspepsia, neurological disease, various degenerative changes in joints, chronic headache, and chronic fatigue syndrome [6]. In order to prevent these problems, physical therapy, exercise therapy, and spine and posture correction methods are applied as therapeutic interventions. Especially in advanced countries like USA and Canada, large number of patients with spine diseases are treated with chiropractic care, and the ones with back pain who are treated with chiropractic care have three times higher satisfaction rate than the ones getting general medical treatments [7]. The principle of chiropractic is to improve motor mechanical functions by maintaining the spine and pelvis in the optimum condition [8]. Chiropractic changes the motility of the spine by correcting abnormal spinal segments and removes neural compression, and the normal efferent neural activity nurtures the ability to heal oneself. However, correction of abnormal spinal segments cannot cure the symptoms completely. To prevent the vertebral bodies that are already displaced from going back to the pre-therapy positions, deep muscles and soft tissues such as ligaments need to be strengthened by exercise therapy so that they find their correct positions 
Table 1 Subject physical characteristics

\begin{tabular}{lcccc}
\hline Classification & Age & Height & Weight & $\begin{array}{c}\text { Percentage } \\
\text { of body fat }\end{array}$ \\
\hline CG $(n=8)$ & $16.4 \pm 1.84$ & $16.4 \pm 1.84$ & $63.8 \pm 1.99$ & $21.5 \pm 2.01$ \\
EG $(n=8)$ & $17.1 \pm 1.52$ & $17.1 \pm 1.52$ & $63.6 \pm 3.17$ & $21.3 \pm 1.57$ \\
\hline
\end{tabular}

and stay firm. Continuous lumbar muscle strengthening and improvements in flexibility decreases spinal deformation, and abdominal and hip muscle strengthening increases intra-abdominal pressure which subsequently decreases loading in intervertebral foramen, thus preventing the back pain caused by scoliosis and its recurrence [9]. Therefore, this study aims to find the effects of chiropractic and lumbar exercise program on lumbar muscle strength and scoliosis improvement in patients with scoliosis and to provide the basic data for health management and rehabilitation program.

\section{Methods}

\subsection{Subjects}

The subjects for this study were recruited from K Clinic in the south area. Written informed consent was obtained from the patient for the publication of this report and any accompanying images. Sixteen adolescents in their growth period, who were diagnosed with scoliosis for having Cobb's angle greater than $10^{\circ}$ on X-ray but did not need surgery, were recruited. The subjects were divided into two groups: half in chiropractic with lumbar exercise program (EG: exercise and chiropractic group, $n=8$ ) and the other half in chiropractic only group (CG: chiropractic group, $n=8$ ). The physical characteristics of the subjects are shown in Table 1.

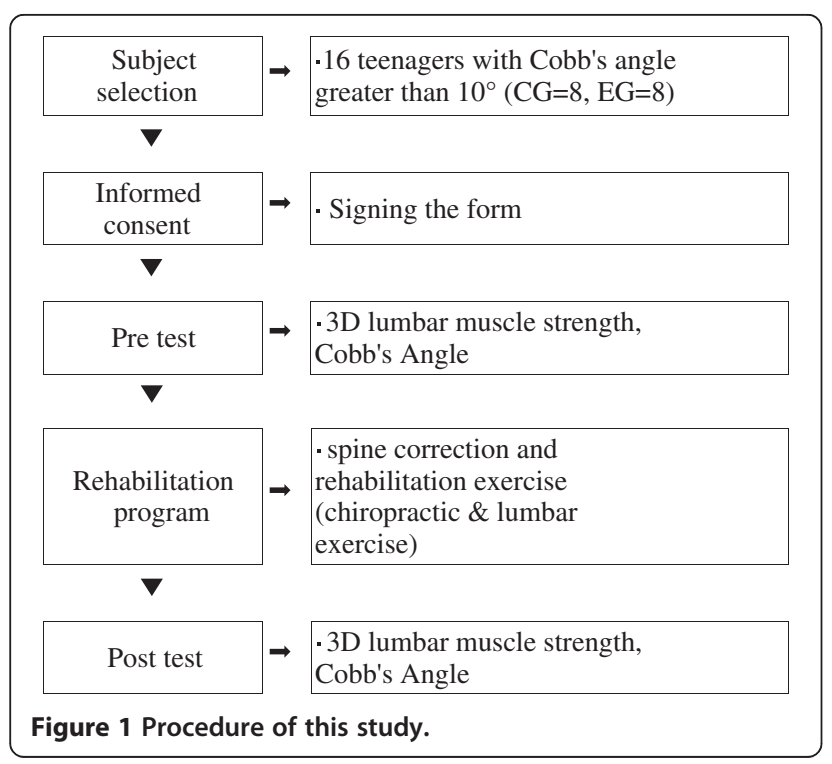

Table 2 Item of inspection and measurement equipment

\begin{tabular}{lcc}
\hline Item of inspection & $\begin{array}{c}\text { Measurement } \\
\text { equipment }\end{array}$ & Manufacturer \\
\hline Height and weight & DS-102 & $\begin{array}{c}\text { DONG SAHN JENIS } \\
\text { Co., Ltd. (KOREA) }\end{array}$ \\
Percentage of body fat & Inbody 330 & $\begin{array}{c}\text { Biospace Co., } \\
\text { Ltd. (KOREA) }\end{array}$ \\
Cobb's angle (X-ray) & DKII525R & CASCO, Co., Ltd. (KOREA) \\
3D lumbar muscle strength & CENTAUR & Biofeedback Motor \\
& 3D system & Control (Germany) \\
\hline
\end{tabular}

\subsection{Study procedure}

The detailed study procedure of this study is shown in Figure 1.

\subsection{Measurements and methods}

The measurements in this study are shown in Table 2.

\subsubsection{D spinal muscle strength device}

CENTAUR (CENTAUR 3D system, Biofeedback Motor Control, Germany) was used to measure normal lumbar muscle strength. As shown in Figure 2, the spinal muscle strength was measured by categorizing the horizontal plane into $0^{\circ}, 90^{\circ}, 180^{\circ}$, and $-90^{\circ}$, tilting up to $90^{\circ}$ vertically.

As the measurement method is done, the examiner stood up on the 3D spinal exercise device, placed both hands on the opposite side of the shoulders, placed his feet on the center of the footplate, and contracted transversus abdominis muscle to maintain basic tension. The overall spine was to be maintained in neutral position, and the examiner tilted the plane $90^{\circ}$ vertically

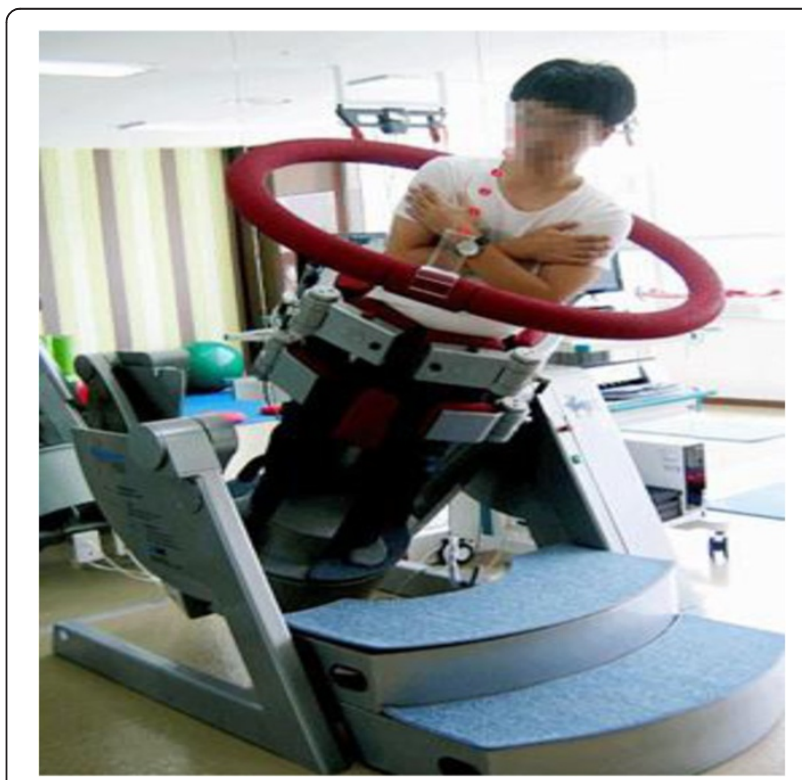

Figure 2 Picture of 3D lumbar muscle strength measurement. 


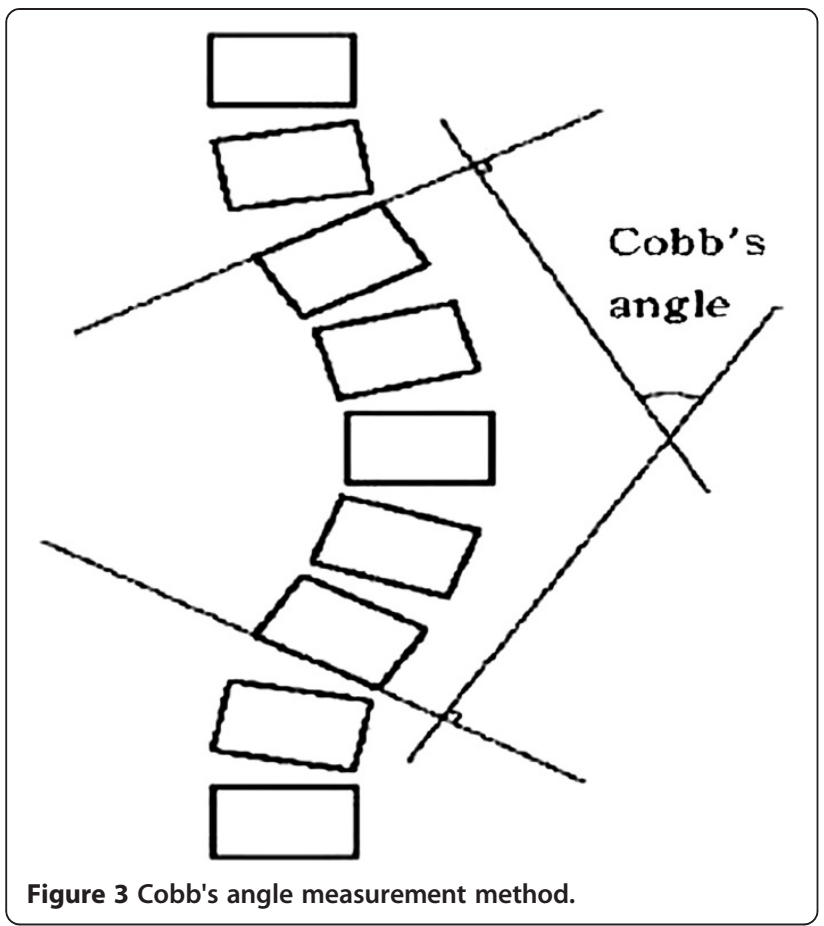

and stopped when the subject could not maintain neutral position. The muscle strength in each of the four directions on the horizontal plane was measured before and after exercise.

\subsubsection{Cobb's angle}

Cobb's angle of the subjects was measured from an X-ray. Cobb's angle is determined on the superior and interior curve to be measured as shown in Figure 3. One line is drawn on the superior end of spine and the other line on the inferior end of the spine. Perpendicular lines to each
Table 4 Changes in pre-post muscle strength (means \pm SD)

\begin{tabular}{lcccc}
\hline Variable & Group & Before $(\mathbf{k N m})$ & After (kNm) & $\Delta \%$ \\
\hline $0^{\circ}$ & CG & $61.4 \pm 17.56$ & $70.0 \pm 16.91$ & 14.0 \\
& EG & $71.3 \pm 13.72$ & $88.4 \pm 13.48$ & 24.0 \\
$90^{\circ}$ & CG & $49.3 \pm 10.73$ & $57.6 \pm 8.44$ & 16.8 \\
& EG & $54.4 \pm 8.72$ & $71.1 \pm 9.41$ & 30.7 \\
$180^{\circ}$ & CG & $32.3 \pm 12.37$ & $38.3 \pm 11.34$ & 18.6 \\
& EG & $35.3 \pm 5.57$ & $52.3 \pm 7.92$ & 48.2 \\
$-90^{\circ}$ & CG & $48.3 \pm 11.90$ & $54.9 \pm 11.35$ & 13.7 \\
& EG & $54.7 \pm 9.12$ & $71.1 \pm 12.33$ & 30.0 \\
\hline
\end{tabular}

$C G$, chriopractic group; $E G$, exercise and chriopractic group.

of these two lines are drawn, and the angle formed by the crossed lines becomes the degree of the curve [9].

\subsection{Rehabilitation program}

The detailed contents of the rehabilitation program in this study are shown in Table 3. Chiropractic treatment was to be safely performed by a medical doctor (MD and DC, Doctor of Chiropractic); deformation angle was confirmed from an X-ray, and correction for lumbar and pelvic dislocation was performed three times a week for 12 weeks.

\subsection{Data analysis}

For this study, SPSS 20.0 for Windows was used for statistic analysis. ANOVA was used to find out the difference between groups and between times in every variable. Statistical significance was set at $p=0.53$.

\section{Results}

\subsection{Changes in lumbar muscle strength}

The results of pre- and post-treatment measurement average and standard deviation in each group on lumbar

Table 3 Rehabilitation program

\begin{tabular}{|c|c|c|c|c|c|}
\hline \multicolumn{2}{|l|}{ Treatments } & \multirow{2}{*}{$\begin{array}{l}\text { Forms } \\
\text { Stationary bicycle }\end{array}$} & \multirow{2}{*}{$\begin{array}{c}\text { Frequency } \\
\text { 3/week }\end{array}$} & \multirow{2}{*}{$\begin{array}{c}\text { Set } \\
1\end{array}$} & \multirow{2}{*}{$\frac{\text { Treatment time }}{5 \mathrm{~min}}$} \\
\hline Lumbar exercise program & Warm up ${ }^{a}$ & & & & \\
\hline & Stretching ${ }^{\mathrm{b}}$ & $\begin{array}{l}\text { Static/dynamic stretching (iliopsoas m/piriformis } \\
\mathrm{m} / \text { hamstring } \mathrm{m} / \text { trunk twist) }\end{array}$ & 3/week & 5 to $10 s / 5$ times & $10 \mathrm{~min}$ \\
\hline & Main exercise & Dumbbell side bending exercise & 3/week & 5 times (10-s hold)/3sets & $20 \mathrm{~min}$ \\
\hline & & Thoracic lateral flexion exercise & & & \\
\hline & & Trunk extension exercise & & & \\
\hline & & Scapula stabilizing exercise & & & \\
\hline & & Lumbar stabilizing exercise & & & \\
\hline & Cool down ${ }^{a}$ & Stationary bicycle & 3/week & 1 & $5 \mathrm{~min}$ \\
\hline \multirow[t]{4}{*}{ Chiropractic } & & Cervical spine lateral correction (left and right) & 3/week & 1 & $10 \mathrm{~min}$ \\
\hline & & Thoracic spine rotation correction in sitting & & & \\
\hline & & Lumbar spine rotation correction in supine & & & \\
\hline & & Pelvic, prone, and posteroinferior correction & & & \\
\hline
\end{tabular}

${ }^{a}$ Saddle height at anterior superior iliac spine, knee flexion kept at $5^{\circ}$ to $10^{\circ}, 50 \mathrm{~W}, 60 \mathrm{rpm}$. ${ }^{\mathrm{b}}$ Stretching within painless and no-hyperflexion ranges. 
Table 5 Results of two-way repeated ANOVA on peak torque of $0^{\circ}$

\begin{tabular}{lccccc}
\hline Source & SS & MS & $\boldsymbol{d f}$ & $\mathbf{F}$ & $\boldsymbol{p}$ value \\
\hline Group & 700.07 & 700.07 & 1 & 3.05 & 0.106 \\
Error & 277.28 & 23.10 & 12 & 229.48 & \\
Time & $1,157.14$ & 115.14 & 1 & 50.07 & 0.001 \\
Time $\times$ Group & 128.57 & 128.57 & 1 & 5.56 & 0.036 \\
Error & 277.28 & 23.10 & 12 & & \\
\hline
\end{tabular}

muscle strength after dividing 16 subjects into chiropractic with exercise group (EG, $n=8$ ) and chiropractic only group (CG, $n=8$ ) are shown in Table 4 . Enhanced improvement in overall lumbar muscle strength in each measurement angle $\left(0^{\circ}\right.$ to $\left.-90^{\circ}\right)$ was observed in EG when compared to CG. As shown in Table 4, ' $\Delta \%$ ' symbol refers to the amount of change between the time (pre and post).

\subsubsection{The $0^{\circ}$ change in lumbar muscle strength}

As shown in Table 5, interaction effects were found in between-group and measurement time difference $(p<0.05)$. A significant difference was noted in temporal difference $(p<0.05)$, but between-group difference was not significant.

\subsubsection{The $90^{\circ}$ change in lumbar muscle strength}

As shown in Table 6, interaction effects were found in between-group and measurement time difference $(p<0.05)$. A significant difference was revealed in temporal difference $(p<0.01)$. But, between-group difference was not significant.

\subsubsection{The $180^{\circ}$ change in lumbar muscle strength}

As shown in Table 7, interaction effects were found in between-group and measurement time difference $(p<0.01)$. A significant difference was observed in temporal difference $(p<0.01)$. However, between-group difference was not significant.

\subsubsection{The $-90^{\circ}$ change in lumbar muscle strength}

As shown in Table 8, interaction effects were found in between-group and measurement time difference

Table 6 Results of two-way repeated ANOVA on peak torque of $90^{\circ}$

\begin{tabular}{lccccc}
\hline Source & SS & MS & $\boldsymbol{d f}$ & $\mathbf{F}$ & $\boldsymbol{p}$ value \\
\hline Group & 306.446 & 306.446 & 1 & 3.727 & 0.078 \\
Error & 986.643 & 82.220 & 12 & & \\
Time & $1,093.750$ & $1,093.750$ & 1 & 99.110 & 0.001 \\
Time $\times$ Group & 124.321 & 124.321 & 1 & 11.265 & 0.006 \\
Error & 132.429 & 11.036 & 12 & & \\
\hline
\end{tabular}

Table 7 Results of two-way repeated ANOVA on peak torque of $180^{\circ}$

\begin{tabular}{lccccc}
\hline Source & SS & MS & $\boldsymbol{d f}$ & $\mathbf{F}$ & $\boldsymbol{p}$ value \\
\hline Group & 236.046 & 236.046 & 1 & 2.613 & 0.134 \\
Error & 993.762 & 90.342 & 12 & & \\
Time & 854.538 & 854.538 & 1 & 55.294 & 0.001 \\
Time $\times$ Group & 195.462 & 195.462 & 1 & 12.648 & 0.005 \\
Error & 170.000 & 15.455 & 11 & & \\
\hline
\end{tabular}

$(p<0.05)$. A significant difference was seen in temporal difference $(p<0.01)$, but between-group difference was not significant.

\subsection{Changes in Cobb's angle}

The results of pre- and post-treatment measurement average and standard deviation in each group on lumbar muscle strength after dividing 16 subjects into chiropractic with exercise group (EG, $n=8$ ) and chiropractic only group (CG, $n=8$ ) are shown in Table 9. As can be seen in Table 9, Cobb's angle was more improved in EG when compared to CG.

As shown in Table 10, interaction effects were found in between-group and measurement time difference $(p<0.01)$. A significant difference was observed in both temporal difference and between-group difference $(p<0.05)$.

\section{Discussions}

Spine can be seen as a single body since the cervical spine, thoracic spine, lumbar spine, and pelvic bones are in segments. Pelvic symphysis is composed of hip joint, sacroiliac joint, and pubic symphysis, which cooperate in stabilizing and mobilizing the body. The ultimate goal of rehabilitation therapy for the patients with scoliosis is to prevent various complications that can be developed by scoliosis and to improve the quality of life. Spinal instability not only causes back pain but also difficulty in functional movements and maintaining postures; therefore, strengthening the deep muscles of the spine and lumbar spine area is important in securing stability [10]. Therefore, it is proposed that applying the

Table 8 Results of two-way repeated ANOVA on peak torque of $-90^{\circ}$

\begin{tabular}{lccccc}
\hline Source & SS & MS & $\boldsymbol{d f}$ & $\mathbf{F}$ & $\boldsymbol{p}$ value \\
\hline Group & 451.446 & 451.446 & 1 & 3.857 & 0.073 \\
Error & $1,404.429$ & 117.036 & 12 & & \\
Time & 925.750 & 925.750 & 1 & 49.217 & 0.001 \\
Time $\times$ Group & 170.036 & 170.036 & 1 & 9.040 & 0.011 \\
Error & 225.714 & 18.810 & 12 & & \\
\hline
\end{tabular}


Table 9 Experiment before and after the Cobb's angle change (means \pm SD)

\begin{tabular}{lcccc}
\hline Variable & Group & Before $\left(^{\circ}\right)$ & After $\left(^{\circ}\right)$ & $\Delta \%$ \\
\hline Cobb's angle & CG & $24.4 \pm 6.06$ & $19.9 \pm 6.28$ & 18.4 \\
& EG & $19.1 \pm 7.97$ & $8.6 \pm 7.04$ & 55.0 \\
\hline
\end{tabular}

$C G$, chriopractic group; $E G$, exercise and chriopractic group.

rehabilitation therapy that is suggested in this study can be meaningful.

Studies on rehabilitation exercise for scoliosis treatment have reported that teenagers in their growth period can prevent scoliosis by performing spine and pelvic muscle strengthening exercises, and these exercises can prevent the progression of scoliosis [11]. Kim et al. [12] reported that combining exercise therapy and Chuna manual treatment for scoliosis was effective, and furthermore, exercise therapy showed better results than Chuna manual treatment. The study by Farady [13] showed that exercise is important for scoliosis treatment because it corrects or prevents scoliosis and is very effective when combined with other treatments for increasing the effects of orthosis. When passive or active exercises including posture correction exercise, spine stretching exercise, and muscle strengthening exercise are performed several times a day, the overall efficacy can be seen on scoliosis, and this observation supports the study that reported close relationship between exercise, ligament and muscle fascia condition, and joint elasticity [14]. Thus, exercise can affect overall movements of the cartilage tissues around joints. This observation corresponds to Kisner's theory [15] that lumbar stabilizing exercise increases range of motion by recovering the functions of deep abdominal muscles and stabilizing muscles, which contribute to trunk stabilization. On the other hand, Wiss [16] reported increase in lung capacity in scoliosis patients, who received exercise therapy. This report shows that exercise is not only important for the spine but also for the functional abilities of the body. The effective results of the present study are in correspondence with the previous studies. Hence, it is appropriate to state that combined chiropractic and exercise therapy serves as a correction therapy for scoliosis patients. In other words, this study is meaningful in suggesting the possibility of chiropractic and lumbar exercise in correcting the dislocated body

Table 10 Results of two-way repeated ANOVA on Cobb's angle

\begin{tabular}{lccccc}
\hline Source & SS & MS & $\boldsymbol{d f}$ & $\mathbf{F}$ & $\boldsymbol{p}$ value \\
\hline Group & 240.286 & 240.286 & 1 & 5.421 & 0.038 \\
Error & 531.946 & 44.329 & 12 & & \\
Time & 393.750 & 393.750 & 1 & 66.316 & 0.001 \\
Time $\times$ Group & 63.000 & 63.000 & 1 & 10.611 & 0.007 \\
Error & 71.250 & 5.937 & 12 & & \\
\hline
\end{tabular}

parts and returning them to normal locations. The 12-week rehabilitation program of this study can be interpreted as an effective treatment method in correcting the spine with chiropractic only, but the effects are greater when it is combined with exercise.

\section{Conclusions}

In conclusion, the present study aimed to test the effectiveness of the program on lumbar muscle strength and Cobb's angle by dividing 16 scoliosis subjects into chiropractic only group (CG: chiropractic group, $n=8$ ) and chiropractic and exercise group (EG: exercise and chiropractic group, $n=8$ ) to perform the program for 12 weeks. The results showed significant increase in lumbar muscle strength $(p<0.05)$ in both CG and EG. However, between-group difference was not found, but EG showed higher improvement. Cobb's angle was significantly increased in CG and EG pre- and post-experiment, and between-group difference was also significant $(p<0.05)$.

Therefore, combining lumbar exercise and chiropractic program for scoliosis patients can be a more effective rehabilitation therapy than applying chiropractic care only.

\section{Competing interests}

The authors declare that they have no competing interests.

\section{Author details}

${ }^{1}$ Department of Health Administration, Dongshin University, 252 Daehodong, Naju, Jeonnam 520-714, Republic of Korea. ${ }^{2}$ Department of Sports Science, Sunmoon University, Kalsan-ri, Tangjeong-myeon, Asan-si, Chungnam 336-708, Republic of Korea. ${ }^{3}$ Division of Computer Engineering, Mokwon University, 88 Doanbuk-ro, Seo-gu, Daejeon302-729, Republic of Korea. ${ }^{4}$ Department of Exercise Prescription, Dongshin University, 252 Daehodong, NajuJeonnam 520-714, Republic of Korea.

Received: 28 March 2013 Accepted: 30 April 2013

Published: 17 May 2013

\section{References}

1. EM John, JK Clive, Back Pain \& Spinal Manipulation, 2nd edn. (Butterworth-Heinemann, 1997), pp. 13-21

2. WP Bunnell, The natural history of idiopathic scoliosis before skeletal maturity. Spine 11, 773-776 (1986)

3. DS Bradford, Textbook of Scoliosis and Other Spinal Deformities (W. B. Saunders, Philadelphia, 1994), pp. 219-251

4. PD Barnes, TY Poussaint, RL Robertson, Imaging of the pediatric spine and spinal neuraxis. Spine: state of the art reviews (Hanley and Belfus, Philadelphia, 1995), pp. 73-92

5. R Pauschert, F Niethard, Ergebnisse der krankengymnastischen Behandlung auf neurophysiologischer Grundlage bei idiopathischer Skoliose: Eine prospektive Analse, in Wirbelsäulendeformitäten Band 3, ed. by HR Weiss (Stuttgart, Springer, 1994), pp. 47-51

6. DC Craig Liebenson, Rehabilitation of the Spine: A Practitioner's Manual, 2nd edn. (Lippincott Williams \& Wilkins, Baltimore, 2007), p. 136

7. JM Cox, Low back pain: Mechanism, Diagnosis, and Treatment, 7th edn. (Lippincott Williams \& Wilkins, Baltimore, 2002)

8. R Bulbulian, J Burke, JD Dishman, Spinal reflex excitability changes after lumbar spine passive flexion mobilization. J. Manipulative Physiol. Ther. 25(8), 526-532 (2002)

9. R Cailliet, Low Back Pain Syndrome, 5th edn. (FA Davis Co., Philadelphia, 1994)

10. AH Julie, AJ Gwendolen, Long-term effects of specific stabilizing exercise for first-episode low back pain. Spine 26, 243-248 (2001)

11. B Wnuk, J Frackiewicz, J Durmala, K Czernicki, K Wadolowski, Short-term effects of combination of several physiotherapy methods on the respiratory 
function - a case report of adolescent idiopathic scoliosis. Stud. Health Technol. Inform. 176, 402-406 (2012)

12. JJ Kim, JM Lee, JH Shin, The effect of exercise on the LBP \& scoliosis treatment after the TuiNa care. Korea J Sport Sci 14(2), 777-787 (2002)

13. JA Farady, Current principles in nonoperative management of structural adolescent idiopathic scoliosis. Phys Ther 63, 512-523 (1983)

14. T Ritvanen, N Zaproudina, M Nissen, V Leinonen, O Hänninen, Dynamic surface electromyographic responses in chronic low back pain treated by traditional bone setting and conventional physical therapy. J. Manipulative Physiol. Ther. 30(1), 31-37 (2007)

15. C Kisner, LA Colby, Therapeutic Exercise: Foundation and Techniques, 4th edn. (FA Davis, Philadelphia, 2002)

16. HR Wiss, The effect of an exercise program on vital capacity and rib mobility in patients with idiopathic scoliosis. Spine 16, 88-93 (1991)

doi:10.1186/1687-1499-2013-132

Cite this article as: Cheon et al:: Effect of chiropractic and lumbar exercise program on lumbar muscle strength and Cobb's angle in patients with scoliosis for u-Healthcare. EURASIP Journal on Wireless Communications and Networking 2013 2013:132

\section{Submit your manuscript to a SpringerOpen ${ }^{\circ}$} journal and benefit from:

- Convenient online submission

- Rigorous peer review

- Immediate publication on acceptance

- Open access: articles freely available online

- High visibility within the field

- Retaining the copyright to your article 